\title{
La promotion du berbère en Algérie
}

De la prise de conscience intellectuelle au projet de société citoyenne

The Promotion of Berber in Algeria. From Intellectual Awareness to the Civic Society Project.

\section{Chérif Sini}

\section{OpenEdition \\ Journals}

Édition électronique

URL : http://journals.openedition.org/etudesafricaines/18183

DOI : 10.4000/etudesafricaines. 18183

ISSN : $1777-5353$

Éditeur

Éditions de l'EHESS

\section{Édition imprimée}

Date de publication : 5 octobre 2015

Pagination : 445-466

ISSN : 0008-0055

Référence électronique

Chérif Sini, « La promotion du berbère en Algérie », Cahiers d'études africaines [En ligne], 219 | 2015, mis en ligne le 01 janvier 2015, consulté le 01 mai 2019. URL : http://journals.openedition.org/ etudesafricaines/18183; DOI : 10.4000/etudesafricaines.18183 


\title{
La promotion du berbère en Algérie
}

\author{
De la prise de conscience intellectuelle \\ au projet de société citoyenne
}

L'examen sociohistorique et sociolinguistique du parcours de la promotion du berbère en Algérie, depuis la fin du XIX ${ }^{\mathrm{e}}$ siècle à ce jour, touche à l'anthropologie et à l'histoire politique contemporaine de ce pays. Tout a commencé par la (re)valorisation de la langue et de la culture berbères par les premiers lettrés autochtones formés à l'école française, durant la colonisation. Refoulée en raison de l'exigence nationaliste indépendantiste de l'Algérie combattante, la particularité berbère ré-émerge après l'indépendance en opposition frontale à l'arabisation. En dehors des circuits institutionnels de l'Etat et en rupture avec l'orientation idéologique de ce dernier, ses défenseurs kabyles travaillent, pour certains, à l'éveil des consciences sur la menace qui pèse sur leur identité linguistique et culturelle et, pour d'autres, à l'émergence d'une culture berbère écrite tournée vers l'avenir et aspirant à la modernité. Comment donc ces derniers ont-ils procédé pour parvenir à faire du kabyle la langue berbère en voie de devenir le référent national, voire transnational aux autres berbérophones ? Comment s'y sont-ils pris face à l'ostracisme de la politique d'arabisation? En quoi le berbère constitue-t-il l'enjeu central et le moteur du projet de société citoyenne actuellement en devenir en Algérie ?

\section{Les précurseurs}

L'hypothèse selon laquelle les premiers défenseurs du berbère dans le milieu kabyle seraient les instituteurs et lettrés formés à l'école française est plausible. Auteurs de monographies, d'essais de littérature berbère de Kabylie et romancière, Cid Kaoui, Saïd Boulifa, Ben Sdira, Bélaïd Nat Ali, etc., et plus tard Jean Mouhoub Amrouche, Margueritte Taous Amrouche, Mouloud Féraoun, Mouloud Mammeri, Nabil Farès... sont les précurseurs de la prise de conscience du danger qui menace l'existence identitaire, culturelle et linguistique des Kabyles et de l'urgence de fixer/valoriser leur langue. Leurs travaux et/ou œuvres, bien que rédigés en français, traitent du quotidien des populations dont ils sont issus. Beaucoup pourrait être écrit au sujet de leur cadre théorique, influencé par les descriptions européennes de la vie des 
« indigènes ». Leur mérite réside à la fois dans leur « révolte » contre le regard exotique de l'Européen de la littérature (orale) et de l'art kabyles et dans leur engagement pour les droits et les valeurs humanistes. Les idéaux humanistes que l'université et l'école françaises diffusent à propos de la révolution de 1789 trouveront un écho retentissant parmi ces Algériens et les conduiront à la revalorisation de soi en suscitant en eux la curiosité de fouiller dans les écrits traitant du passé nord-africain. Ces lectures leur feront découvrir le passé antéislamique avec son armada d'épopées de royaumes, de héros et résistants berbères. Elles leur feront faire connaissance avec de grands littéraires ou théologiens comme Fronton, Tertullien, Saint Augustin, Saint Optat, Arnaud, Apulée, Saint Cyprien, Lactance... connus et reconnus dans et par la civilisation judéo-chrétienne. À ce sujet, M. Mammeri (1987 : 95) écrit : «Fronton était berbère, Lactance était berbère [...]. Apulée était berbère, et naturellement le plus grand de tous Saint Augustin était berbère. » Ces éléments étaient d'un impact décisif sur le type de représentations mentales que construisaient ces instituteurs autour de leur personnalité, de leur passé et surtout de leur avenir qu'ils diffusaient dans leurs écrits : «L'Eternel Jugurtha » de J. M. Amrouche en est un exemple. Ainsi, l'image de «l'indigène barbare » est petit à petit remplacée par celle valorisante, celle d'une nation très ancienne et résistante à Rome, à Byzance, aux Arabes, aux Français...

Tout en rendant hommage aux premiers missionnaires et descripteurs européens (militaires, ethnologues, historiens, linguistes...), ils se sont démarqués d'eux en s'investissant avec passion dans leur objet. L'exemple le plus connu est celui de S. Boulifa, kabyle, instituteur et premier diplômé du brevet de langue kabyle créé en 1885 à l'École normale supérieure de Bouzaréat, sur les hauteurs d'Alger (Chaker 1991 : 46). Ses travaux, notamment son Recueil de poésie kabyle (Boulifat 1904), sa Méthode de langue kabyle (ibid. 18971913) et d'autres réflexions ethno-historiques s'apparentent à une "défense et illustration de la langue kabyle » (ibid. : 14).

Leur action est strictement d'ordre intellectuel. Mais son impact sur les générations suivantes est déterminant : ces instituteurs-auteurs deviendront en effet des précurseurs et serviront de référence (Chaker 1989-1990: 21). De cette génération datent, en fait, les premiers pas du passage à l'écrit du kabyle. En écrivant sa Méthode de langue kabyle directement en kabyle et avec les caractères communément appelés latins actuellement dans le milieu berbérisant, S. Boulifa venait, d'une part, d'offrir aux Kabyles de sa génération ainsi qu'aux futurs militants kabyles la preuve que, comme le français et l'arabe, « le berbère ça s'écrit » (ibid. : 49) et, d'autre part, d'enclencher un mouvement qui se poursuivra tout au long du $\mathrm{XX}^{\mathrm{e}}$ siècle : celui de l'adoption/ adaptation de l'écriture latine. Il apparaît difficile de saisir les raisons de ce choix, parce que S. Boulifa ne connaissait pas le tifinagh, l'alphabet originel des Berbères ? Parce que matériellement il n'était pas possible de publier en dehors de la graphie latine ? Parce que des Européens dans leurs descriptions avaient déjà utilisé ces caractères et que S. Boulifa n'avait fait qu'adopter 
leur choix ? La dernière hypothèse paraît la plus vraisemblable et la formation francophone de $\mathrm{S}$. Boulifa et de ceux à qui il voulait s'adresser pourrait y être pour quelque chose.

Cette tendance, depuis les publications de Venture de Paradis (1844), de A. Hanoteau (1858, 1867, 1896), etc., jusqu'à l'adoption de ces caractères prononcés «à la française », tout en observant certaines règles de l'orthographe française concernant, par exemple, les affriquées et les sons complexes en général, se poursuivra avec de plus en plus de rigueur dans l'adaptation scientifique liée aux spécificités kabyles. Elle sera renforcée par les publications du Fichier de documentation berbère (1946-1976), par celles des berbérisants européens comme A. Basset, L. Galand, P. PernetGaland, J. Delheur, K. G. Prasse, J. R. Appelgate, etc., et par la génération postindépendance de kabylisants. C'est pourquoi l'initiative durant les années 1930-1940 de Mohand Amokrane Khelifati, auteur d'un alphabet tifinagh adapté au kabyle, ne séduira pas et la remise à l'honneur des tifinagh par l'Académie berbère de Paris $^{1}$ (1966-1978) sera d'un usage plus symbolique, déterminant de la prise de conscience identitaire parmi les jeunes générations, il est vrai, que producteur d'œuvres littéraires ou autres. Car ceux qui écriront en kabyle le feront avec les caractères latins.

\section{Les écrivains kabyles de langue française}

Célèbres pour leurs publications en français, M. Feraoun, J. M. et M. T. Amrouche, M. Mammeri, etc., pour ne citer que les plus connus des écrivains kabyles d'avant l'Indépendance, comptent parmi ceux pour qui la question de la mémoire collective mais aussi celle des identités linguistiques et culturelles kabyles ont constitué le noyau central de leur création poétique et/ou romanesque. Quel que soit le degré de fiction de cette littérature, les paysages des espaces romanesques comme les caractères physiques et moraux des personnages créés cachent mal leur ancrage dans le milieu et pays kabyles. En décrivant, dans leurs romans, la vie quotidienne kabyle, ces auteurs affirmaient leur kabylité en réaction à la tendance assimilationniste de S. El Koubi, M. Talbi, M. Ould Cheikh, R. Zenati, C. Kadi, C. Khodja, etc., auteurs algériens de langue française de la première génération.

Dans ce sillage, s'inscrivaient les traductions en français que J. M. Amrouche a faites de la poésie kabyle en 1939. Publiée à l'attention du public cultivé francophone, cette poésie sort du cadre oral, acquiert une notoriété internationale et remet en cause l'idée souvent négative et méprisante répandue à propos de la littérature berbère : «Il est à peine besoin

1. Fondée par des militants issus de la classe ouvrière, c'est en fait une association de défense de la berbérité dans une logique de rejet de l'arabité et en opposition frontale avec la politique d'arabisation au Maghreb en général et en Algérie en particulier, l'essentiel de ses membres étant Kabyles. 
de dire », écrit A. Hanoteau (1867 : préface), «qu'on ne doit pas s'attendre à rencontrer chez eux [les Berbères] une littérature rappelant, même de loin, celle des nations civilisées ».

Comme pour répondre à A. Hanoteau (1867 : préface [repris par Basset 1920]) qui juge le patrimoine littéraire d'expression kabyle «pauvre, primitif, frustre, répétitif, servile par rapport aux modèles arabo-islamiques », M. Feraoun, connu surtout pour sa production romanesque en français (1950, 1953, 1957), a publié en 1960, aux Éditions de Minuit, Les poèmes de Si Mohand, dans les deux versions, française et kabyle.

Dès son premier texte, La société berbère persiste mais ne résiste pas, publié en 1938², M. Mammeri (1991: 16) s'attaque à l'égalitarisme des Berbères : la raison pour laquelle, selon lui, ces derniers «n'ont jamais formé un État, une civilisation propre à eux » (ibid. : 1) pour asseoir une tradition écrite nécessaire et conséquente à/d'une telle organisation et historicité. La sauvegarde de la culture berbère constitue la préoccupation majeure de son activité intellectuelle : littérature, anthropologie et linguistique. Sans zèle, il parcourait le vaste territoire berbère pour cueillir et fixer, à l'aide des caractères latins, la littérature orale berbère. Les résultats de cette activité sont publiés sous forme de recueils (Cortade \& Mammeri 1967; Mammeri 1969, 1973, 1980a b, c, 1984, 1989), et de contributions dans différentes revues (Aguedal, Revue africaine, Révolution africaine, Reflets, Libyca, Les Temps Modernes, Tafsut, études et débats, Awal, Dérives...). À partir de 1985, la revue Awal, Cahiers d'études berbères dont il est le fondateur, paraîtra régulièrement et servira d'espace d'expression aux berbérisants de différentes nationalités. Son Précis de grammaire berbère, édité par Awal à Paris en 1988 (d'abord publié chez Maspero en 1976 et sous le titre, Tajarrumt $n$ tmazirt, demeure l'œuvre maîtresse de l'activité grammatisante et berbérisante de M. Mammeri. Écrite en caractères latins, cette grammaire servira de base aux jeunes générations d'auteurs kabyles. En revenant souvent dans la mise en discours d'attitudes d'auteurs-locuteurs kabylophones à l'égard des graphies tifinagh, arabe et latine proposées pour le kabyle, le nom de M. Mammeri semble fonctionner comme une référence (Sini 2007), si bien que cette écriture est dite «tamamrit», c'est-à-dire « la mammerienne ». Les étudiants qui assistaient à ses cours de berbère à l'Université d'Alger entre 1965 et 1972, ou qui l'ont connu dans le cadre du Groupe d'études berbères de l'Université Paris 8, deviendront les principaux animateurs de la veine culturaliste kabyle et certains assumeront des responsabilités dans différents appareils politiques à partir de 1988, date à laquelle le multipartisme politique sera toléré en Algérie.

2. Publié en 1938 dans la revue Aguedal, 5, pp. 1-18 et réédité en 1991 dans la revue Culture savante, culture vécue. 


\section{Les militants kabyles du PPA/MTLD}

L'intérêt porté à la fixation-valorisation de la langue et de la culture kabyles par les instituteurs formés à l'école française ne mettra pas longtemps pour germer dans la mouvance nationaliste algérienne naissante, d'abord, dans le milieu ouvrier kabyle en France notamment dans le cadre du Congrès des ouvriers nord-africains de décembre 1924 et, ensuite, dans les organisations à caractère politique et indépendantiste de l'Algérie des années 1930, 1940 et 1950, notamment l'ENA et le PPA/MTLD (l'Étoile nord-africaine 19261937 et le Parti du peuple algérien 1937-1939/Mouvement de triomphe des libertés démocratiques 1946-1954) (Stora 1985; Ouerdane 1990 : 31-44). Le regroupement, au début des années 1930, de militants nationalistes kabyles autour d'Imache Amar et de militants nationalistes essentiellement arabophones autour de Messali El Hadj, pour conduire la direction nationale du PPA, traduit le poids de l'appartenance ethnique et linguistique des deux ensembles en concurrence pour le leadership. «Les références à la tradition berbère sont aussi explicites chez $\mathrm{A}$. Imache que sont celles à l'araboislamisme chez Messali », écrit S. Chaker (1989-1990 : 20). S'il est donc vrai que, durant les années du nationalisme algérien, la communauté des croyants (Harbi 1992 : 28-32) primait sur celle des langues et des cultures, il n'en demeure pas moins que ces dernières étaient plutôt non formulées politiquement mais vécues, et constituait déjà un tabou à taire pour former une nation unifiée à opposer à la France coloniale. Ignorer délibérément l'opposition des Kabyles des années 1840-1850 à reconnaître 1'Emir Abdelkader comme leur chef suprême et commandeur des croyants et ne pas tenir compte du refus des autres régions de l'Algérie de suivre El Mokrani et El Haddad dans leur soulèvement armé en Kabylie de 1871, c'est passer sous silence une réalité sociologique, sociolinguistique et sociopolitique nécessaire à la compréhension du processus de la constitution de la nation algérienne. Ces faits préfiguraient déjà les deux principales tendances qui allaient prédominer dans l'histoire du mouvement indépendantiste algérien et dans celle de l'Algérie indépendante. Dès les premières années du nationalisme algérien, dans le milieu des Oulémas, l'hostilité aux références berbères n'était guère voilée (Desparmet 1938 ; Bousafsaf 1991) : «Quiconque s'éloigne de la langue arabe s'écarte du même coup de l'adoration de Dieu, et quiconque s'écarte de l'adoration de Dieu encourra de sa part un terrible supplice », déclaraient les Oulémas des années 1930 à l'occasion du « Dahir berbère »(Chaker 1989-1990 : 75). En 1948, précise A. Ouerdane (1990 : 72), « les Oulémas réclament la suppression de la chaîne kabyle de radioAlger [mise en place en 1947] et déclarent dans leur organe d'information El-Baçaîr que les Kabyles ne seraient des Algériens à part entière que lorsqu'ils auront cessé de chuchoter ce jargon [la langue kabyle] qui nous écorche les oreilles ».

L'histoire de la principale organisation politique du mouvement nationaliste algérien des années 1930-1940 (le PPA) est émaillé de crises internes 
et successives opposant les militants radicaux et favorables au passage immédiat à l'action armée, majoritairement issus de la Kabylie et se définissant Algériens sans référence culturelle ou linguistique (Ouerdane 1990 : 43), aux partisans de Messali El Hadj, arabophone de retour de son exil du Congo et devenu légaliste en fondant le MTLD et en participant aux élections de l'Assemblée nationale française en novembre 1946 (Harbi 2011: 159, 256, 262, 291 sq.). Ce revirement inattendu de celui auquel s'opposait au début des années 1930 le Kabyle Imache Amar, constitue le début d'un malaise au sein des militants nationalistes partisans de l'action armée, y compris parmi les arabophones de la direction nationale du PPA (Ait Ahmed 1983). C'est aussi durant cette période que Bennaï Ouali, kabyle et membre très actif du PPA, est entré en contact avec un ensemble de lycéens de Ben Aknoun (sur les hauteurs d'Alger), originaires de Kabylie, pour les sensibiliser au patriotisme. Devenus des militants actifs et cadres influents dans le Parti, ces lycéens présentent une particularité qui va les rendre suspects aux yeux de la direction nationale : ce sont des fervents défenseurs de l'action armée. En plus, ils puisent leurs idées révolutionnaires du terroir: leur culture kabyle. En effet, le rapport que présentera Hocine Ait Ahmed, à Zidine- Aîn Defla- en décembre 1948, en qualité de chef de l'os (Organisation spéciale $)^{3}$, dit beaucoup sur l'ancrage dans la tradition révolutionnaire berbère de la vision de ce lycéen, pourtant connu pour ses positions peu tranchées sur la question identitaire berbère même après l'Indépendance, aussi bien de la révolution libératrice que de la future Algérie libre (Harbi 2011 : doc. 1-35). Dans ce climat de malaise et de crise de confiance entre la direction nationale et les militants de la base, notamment ceux de Kabylie, les lycéens de Ben Aknoun font donc leur entrée dans la mouvance nationaliste en lui donnant un cachet révolutionnaire. Euvre de ces lycéens, les premiers chants patriotiques, dont le plus célèbre ekker a mmis umazigh (litt. Debout fils de Berbère) de Mohand Idir Ait Amrane, font leur apparition. Ils sont immédiatement diffusés auprès des masses patriotiques, y compris en dehors de la Kabylie, notamment dans le milieu des scouts musulmans algériens dont Ali Laîmeche, un autre lycéen kabyle, est membre influent, si bien que beaucoup diront plus tard que durant cette période, le nationalisme algérien se chantait en kabyle. Nul doute que cette arrivée dans le PPA/MTLD des A. Laïmeche, H. Ait Ahmed, M. I. Ait Amrane, Omar Oussedik, Amar Ould Hamouda, Saïd Chibane, etc., tous issus de la paysannerie kabyle et de surcroît intellectuels francophones, est pour beaucoup dans l'audace politique des compagnons de Ali Ferhat et Ouali Bennaï à briser le tabou imposé à la dimension berbère de l'identité algérienne par les dirigeants du PPA/MTLD, d'un côté, et dans la peur de voir la direction nationale de la principale organisation indépendantiste dominée par les «berbéristes », de l'autre. La réponse à la demande de la base militante de passer à l'action armée résidera

3. Créée lors du Congrès du Parti tenu en 1947 et tolérée par Messali pour contenir la colère des partisans de l'action armée et opposée au MTLD de Messali. 
dans la création de l'os (chargée de préparer l'insurrection armée). Quant à celle qui a trait à l'intégration de la berbérité parmi les constituants de l'identité algérienne, Messali l'ignore totalement en court-circuitant les tenants de «l'Algérie algérienne » (Ouerdane $1990: 47$ ) dans sa «brochure à l'ONU » où il écrit que «la nation algérienne, arabe et musulmane, existe depuis le $\mathrm{VII}^{\mathrm{e}}$ siècle » faisant fi de la période antéislamique dont les noms de héros et résistants berbères sont mobilisés par les chants patriotiques produits par des militants du PPA/MTLD pour sensibiliser les Algériens à la cause nationale. La réaction des tenants de la ligne de l'Algérie algérienne ne se fera pas attendre : la fédération de France du PPA/MTLD, sous l'impulsion de Rachid Ali Yahia, ami de Ouali Bennaï, vote une motion dénonçant «le mythe d'une Algérie arabo-islamique et défend la thèse de l'Algérie algérienne » (Guenoun 1999 : 21). Le 15 avril 1949 (ibid.), la direction nationale du PPA/MTLD dépêche des commandos pour récupérer avec force les locaux de la fédération de France des mains « scissionnistes », « berbéro-matérialistes » et « alliés du colonialisme ». Depuis, une véritable chasse à l'homme est engagée contre ces militants de l'aile radicale du PPA/MTLD, partisans de l'action armée et d'une Algérie algérienne. Ces militants seront exclus de la direction du Parti et dénigrés dans des campagnes auprès de la base militante (Chaker 1989-1990: 76).

Cet épisode, connu dans la littérature de l'histoire officielle par le vocable de «crise berbériste » est, en fait, antiberbère. Il constitue la première ligne de démarcation entre deux projets de société diamétralement opposés qui traverseront plus au moins «clandestinement» la période postindépendance de l'Algérie pour éclater au grand jour au début des années 1980. Cet épisode, de par l'instrumentalisation des aspirations algérianistes de militants actifs du PPA/MTLD par ses dirigeants nationaux, principalement Messali El Hadj, a marqué de méfiance définitivement les militants kabyles pour qui l'ennemi n'est pas seulement le système colonial mais aussi les tenants de l'arabisme exclusif. Cette crise, à laquelle s'accumuleront d'autres, tout au long de l'histoire de l'Algérie indépendante, jouera un rôle déterminant dans le rejet systématique par les Kabyles de la proposition des caractères arabes pour écrire leur langue. Elle prend fin au début de l'année 1950. Ses répercussions accompagneront aussi bien la révolution algérienne (Ouerdane 1990 : chap. 2), dont les principaux bastions étaient pourtant les monts de la Kabylie et ceux du Chaouia, que la période de la reconstruction du pays : assassinat des Ouali Bennaï, Mbarek Ait Menguellet, Ramdane Abane, etc., durant la révolution de 19541962, marginalisation des intellectuels soupçonnés de sympathie avec le milieu culturaliste kabyle, refus d'expression à tout opposant au projet de société arabo-islamique exclusif prôné par l'équipe au pouvoir et présenté comme étant celui de la nation, interdiction de vente de toute production berbérisante, etc. Cette obsession de l'autorité centrale d'anéantir ce que M. Mammeri appelle « la culture du peuple» pour la remplacer par ce que le même Mammeri appelle «la culture pour le peuple » constitue le contenu idéologique de l'arabisation (Hadjar 1974 ; Salama 1981 ; Cheriet 1983 ; Granguillaume 1983, 2004 ; Moatassime 1992). 


\section{L'arabisation : être ou paraître?}

Ancrée dans le mouvement indépendantiste, l'option pour la culture araboislamique à l'exclusion de tout autre référence culturelle et linguistique est affirmée dans la Charte d'Alger de 1964 qui stipule que «l'Algérie est un pays arabo-musulman ». Cette position est réaffirmée à toutes les occasions par les dirigeants politiques algériens.

À peine l'Indépendance acquise, la Kabylie entre en conflit, dans un premier temps, contre l'armée dite des frontières (Ouerdane 1990 : 109-152) qui instaurera un régime socialiste d'obédience arabo-islamique contre lequel la même Kabylie, cette fois-ci au nom du Front des forces socialistes, entre en rébellion sans succès de septembre 1963 à juin 1965. Les deux échecs successifs de la Kabylie à peser sur l'orientation politique du pays ont renforcé l'isolement des populations kabyles, traitées de «traîtres à la solde du néocolonialisme ». Commence alors l'ère du paraître dictée par l'omerta décrétée par le régime en place. À la recherche du contrôle total de la société et des consciences, celui-ci s'investit dans la destruction de l'héritage colonial et populaire d'expression arabe et surtout berbère. Les premiers secteurs concernés par cette orientation sont ceux de l'éducation et de la justice. Dans une langue pratiquée nulle part, le premier endoctrine, le second condamne. La mission de l'école consiste à former des militants, celle de la justice à montrer la voie à suivre : la soumission au dressage socioculturel collectif (Benrabah 1996). Prêché dans les tribunes politiques officielles et consacré par les textes fondateurs de la République algérienne, le modèle de citoyen algérien est exécuté à l'école par des moniteurs et des instituteurs moyen-orientaux. Ainsi a-t-on imposé le silence aux défenseurs de la culture algérienne d'expression arabophone, francophone et berbérophone : c'est la révolution culturelle! Tout en ratifiant la déclaration des droits de l'Homme, celle des droits des peuples, etc., l'Algérie officielle supprime la chaire de berbère de l'Université d'Alger dès 1962 (Chaker 1991 : 47), fait dissoudre « l'alphabet tifinagh en fonte de l'université d'Alger sur ordre de Ben Bella en personne » (Sadi $1991: 291$ ), fait disparaitre le Fichier de documentation berbère en 1977 (Chaker 1991 : 47), interdit Sliman Azem de diffusion sur les ondes de la radio kabyle d'Alger, interdit la participation à M. T. Amrouche au festival culturel panafricain... La fermeture des voies pour l'expression de l'opposition politique et culturelle sera renforcée par la répression à partir des années 1970, notamment avec la Charte et la Constitution de 1976 et le début de l'arabisation systématique de l'école, de la justice... De l'interdiction de production sur scène, de vente sur le marché de l'édition, etc., on est passé à l'incarcération, parfois à la condamnation à mort, des militants de la berbérité, souvent enfants de martyrs de la révolution algérienne, en les accablant d'accusation de trahison et de complot contre l'État. Depuis, écrit S. Chaker (1989-1990 : 50), «du fait de [ce] contexte culturel et politique, chanter, parler en public, écrire en berbère est en soi un engagement ». 
À partir de 1965, la résistance à la politique d'arabisation s'organisera clandestinement dans le lycée de Tizi-Ouzou où des élèves ont été exclus parce qu'ils ont proposé de jouer une pièce de théâtre en berbère (Redjala 1994) et dans la cité universitaire de Ben Aknoun, à Alger, où résidaient en qualité d'étudiants les principaux futurs animateurs du Printemps berbère de 1980. Cette résistance s'exprimera essentiellement à travers la chanson et le sport. En effet, à partir des années 1970 le disque puis la bande magnétique deviendront les vecteurs de la revendication identitaire berbère et celui du début de l'unification du berbère : d'où la consolidation du sentiment d'appartenance au même groupe linguistique (Chaker 1989-1990 : 42). Si les textes chantés de Sliman Azem, de Lounis Ait Menguellet, de Chérif Kheddam, de Lounes Matoub, etc., tous d'orientation contestataire, se limiteront à la Kabylie et à un degré moindre aux autres régions berbérophones, ceux d'Idir, de Djamel Allam, Taous Amrouche, Imazighen Imula, Djurdjura, Abranis, Mexa... connaîtront un succès international qui fera sortir la revendication kabyle du ghetto de l'enfermement dans lequel l'arabisation l'acculait.

Durant la décennie 1970-1980, le vocable même de « berbère » deviendra un tabou : la centralisation à l'extrême du régime et sa redoutable police politique ne laisseront aucune chance à l'expression de tout particularisme et de toute contestation, de surcroît en provenance de Kabylie.

La même période enregistre la montée en puissance d'une activité culturelle écrite en berbère dans le milieu kabyle en France, largement diffusée en Kabylie et dans l'Algérois par le tissu militant de la cause berbère : traduction vers le kabyle de pièces de théâtre de B. Brecht, W. Shakespeare, etc., et diffusion de bandes dessinées en kabyle, apparition des premiers romans kabyles écrits en caractères latins parfois avec la présence symbolique sur la première page et/ou la dernière page de la couverture des tifinagh, jamais avec les caractères arabes. Cette percée continue à côté de celle de Loucif Hammani en boxe et celle, dominante, du football algérien puis africain de la JSK (Jeunesse sportive de Kabylie), dont les initiales sont révélatrices des motivations identitaires aussi bien du groupe que des supporters et que les autorités politiques remplaceront par JET (Jeunesse électronique de Tizi-Ouzou), mais que le génie populaire traduira par «Jugurtha existe toujours », redonnera de la fierté aux populations kabyles et réduira la portée des échecs de l'été 1962 et de 1963-1964 au point où lors de la finale de la coupe d'Algérie de football, remportée par la JSK, le président d'alors, H. Boumédiène, sera décrié dans la capitale, Alger, par des dizaines de milliers de supporters kabyles à qui on avait distribué le tract suivant: «Les Arabes ont emprunté leur alphabet aux Araméens, les Européens aux Phéniciens, les Berbères, eux, ne doivent rien à personne. Autrement dit, ils sont les inventeurs de leur propre système d'écriture », c'est-à-dire les tifinagh.

L'autre forme de résistance à l'arabisation se traduira par l'adhésion, pour certains, à l'Académie berbère de Paris (1966-1978), connue pour ses positions radicales, à l'image de son principal animateur, Mohand Arab 
Bessaoud, qui, ancien militant nationaliste et combattant pour l'indépendance de l'Algérie, prône la résistance berbère à la domination arabe, et, pour d'autres, au noyau d'universitaires investis dans la production artistique, littéraire et grammatisante animée par l'écrivain et anthropologue M. Mammeri et relayé par le linguiste S. Chaker. Parmi les différences entre ces deux orientations, il faut retenir le choix graphique.

Les tifinagh, aménagés et appelés néo-tifinagh, sont diffusés par les adhérents à l'Académie berbère de Paris qui rejetait à la fois les caractères arabes et les caractères latins. Le choix des tifinagh pour servir de support au berbère durant cette période de répression et de velléité de contrôle idéologique de la société, est dans la logique des animateurs de l'Académie berbère de Paris : au projet mythique de retrouver l'âge d'or de la civilisation arabo-islamique, en imposant l'arabe classique dans tous les domaines de la vie publique, est opposé celui qui consistait à retourner aux valeurs ancestrales que symbolise l'authenticité des tifinagh. C'est donc l'aspect discriminatoire des tifinagh aussi bien à l'égard de l'arabe, symbole du pouvoir central, que du français, langue de l'ex-colonisateur, qui semble dicter ce choix. Ce choix était d'autant plus émotionnel qu'il apportait un argument de taille et revalorisant leur revendication: le berbère, comme les autres langues, voire mieux, possède une écriture. Cette représentation jouera un rôle déterminant dans la prise de conscience identitaire et dans la mobilisation pour la réhabilitation de la berbérité au point où les caractères tifinagh se confondront avec la langue berbère, dans la conscience des militants et sympathisants de la revendication berbère. Et les autorités politiques ne se trompaient pas lorsqu'elles arrêtaient systématiquement les personnes détentrices de cet alphabet (Chaker 1989).

Regroupés à Paris-Vincennes, les berbérisants, préoccupés surtout par l'aménagement du berbère pour en faire un instrument de communication moderne, poursuivent l'adaptation de l'alphabet latin. Cette adaptation se traduira notamment par la réduction des digrammes et trigrammes à une seule lettre, quitte à la suscrire ou à la souscrire d'un point, d'une cédille ou d'un tiret pour rendre les unités fonctionnelles berbères. Ainsi, les traits phonétiques notés dans l'adoption des Ben Sdira, Boulifa, etc., céderont devant la tendance phonologique favorable à l'unification de la langue au moyen des règles d'écriture et de la néologie. Cette démarche sera annoncée puis précisée et adoptée par les utilisateurs à partir de 1980 : à chaque son (fonctionnel) correspond un seul caractère et le même caractère écrit toujours le même son (Achab 1998 : 25).

S'il est vrai que la production de cette activité grammatisante circulera en Algérie par le biais du réseau de militants kabyles, l'adhésion des nouvelles générations à la production avec les caractères latins se fera attendre et, à quelques exceptions, n'arrivera pas à s'imposer durant cette décennie 1970-1980 : les néo-tifinagh, plus connus et plus mobilisateurs, constituaient le symbole de la berbérité... 
Le Printemps 1980: occupation pacifique de la rue et naissance du Mouvement culturel berbère

Ouvert à la fin des années 1970, le centre universitaire de Tizi-Ouzou deviendra aussitôt le centre nerveux d'un mouvement porté d'abord par des universitaires et des cadres des complexes industriels et de l'hôpital de la même ville, puis élargi à l'ensemble de la population kabyle. Ce mouvement était, en fait, en gestation : la contestation, lors des débats sur la Charte nationale de 1976, contre les velléités exclusivement arabisantes de l'Algérie officielle que confirmera la Constitution de 1976 et l'école fondamentale, l'affaire des «berbéristes poseurs de bombes », etc., ont élargi davantage le fossé entre le pouvoir central et les populations kabyles. L'interdiction à M. Mammeri, le 10 mars 1980, de présenter une conférence sur la poésie kabyle ancienne à l'Université qui portera plus tard son nom, était la cause directe de la rupture totale. Après plusieurs jours de grève observée au centre universitaire en signe de protestation contre cette interdiction et sans qu'il y ait de réponse à leur doléance principale, c'est-à-dire l'ouverture d'un débat sur la culture nationale et le droit d'expression, les étudiants et les enseignants décident de sortir dans la rue, bravant ainsi l'interdiction de marcher... Outre les revendications culturelles réclamées, des slogans hostiles au régime ont été scandés. Les autorités décident de mettre à exécution leur menace : les cités universitaires sont investies et les étudiants maltraités. Les villageois avoisinants descendent occuper les rues de la ville de Tizi-Ouzou en guise de mécontentement et de solidarité avec les victimes de la répression. Les affrontements avec les forces de l'ordre se généraliseront à toute la Kabylie et atteindront l'Algérois. Aucun bilan humain n'a été porté à la connaissance du public, si bien que les spéculations ont fait état de plusieurs morts (Ait Sahlia 1999: 72). C'est dans ce contexte que, pour canaliser la contestation, un collectif de militants décide de lancer le MCB, Mouvement culturel berbère (Ait Larbi 2011).

Malgré la crédibilité et l'audience internationales dont bénéficie ce dernier, grâce au réseau tissé par ses militants dans l'immigration, malgré son caractère pacifique et ses revendications portés par l'ensemble de la population kabyle, les dirigeants algériens persistent, d'un côté, à réprimer et à déclarer tantôt «le berbère est une création coloniale » ou «le berbérisme est la doctrine d'un groupuscule d'intellectuels inféodés au néocolonialisme » et, tantôt, «le berbère n'est pas une langue parce qu'il n'a pas de grammaire » ou «parce qu'il n'a pas d'écriture », ou encore «le berbère est un dialecte arabe et les Berbères sont d'origine arabe» (Chaker 1989-1990 : 67-77). De l'autre côté, la répression de ce mouvement ayant échoué à l'étouffer en Kabylie, l'anathème jeté sur ses animateurs ne les ayant pas discrédités, car ce sont souvent des enfants de martyrs et d'anciens combattants pour l'indépendance de l'Algérie, les autorités centrales promettent un débat sur la culture populaire et sa prise en charge par l'État. Ce débat n'aura pas lieu entre les dirigeants politiques partisans de «la culture pour le peuple» 
(Mammeri 1991 : 136) et les défenseurs de la «culture du peuple» (ibid.) comme promis, mais entre les clans partageant le pouvoir. Deux grandes tendances, en apparence, se dégagent, alors que les arabisants sont ouvertement pour l'exclusion de la berbérité, les francisants préfèrent sa marginalisation pour éviter ainsi d'affronter ses militants (Chaker 1981: 457).

Le FLN $^{4}$, parti-État, décide, depuis cette date, d'une part, d'accélérer l'arabisation (ibid. 1989-1990: 32) et, d'autre part, d'ouvrir une filière de culture populaire rattachée au département d'arabe à l'Université d'Alger et à l'Université de Tlemcen, loin des réalités berbérophones (essentiellement de Tizi-Ouzou et de Bejaïa). Naturellement ces décisions ont exacerbé la frustration et renforcé la détermination kabyle : à toutes les occasions, les militants de la berbérité (se) manifestent avec de plus en plus de sérénité et d'organisation; les stades de football du $1^{\mathrm{er}}$ novembre de Tizi-Ouzou et du 5 juillet d'Alger étaient le lieu de diffusion rapide de tracts appelant à faire grève, à marcher. Très souvent, il suffisait d'une information de bouche à oreille pour que la rencontre de football soit suivie d'une marche au rythme des chants d'Imazighen Imula, d'Ait Menguellet, de Matoub... Ces marches finissaient souvent dans la confrontation avec les forces de l'ordre.

En guise de réponse officielle à cette mobilisation, le président Chadli déclare dans son discours au $5^{\mathrm{e}}$ congrès du FLN, tenu en décembre 1983: «La révolution algérienne est une révolution nationaliste, islamique dans son esprit, socialiste dans sa démarche et arabe dans son expression. [...] Quant à la langue, l'appartenance de l'Algérie à la nation arabe et la contribution conséquente du peuple algérien à l'épanouissement de la prestigieuse civilisation arabo-islamique, plaident, à eux seuls, pour l'arabe comme moyen d'expression et véhicule culturel. [...] Arabisation, terme impropre s'il en est, car on n'arabise pas des Arabes, ou tout au moins un peuple qui s'identifie à la nation arabe et qui en est partie prenante $»^{5}$.

Ce discours prélude, en fait, la charte nationale (amendée) de 1986 qui stipule à la page 109 que «l'histoire de l'Algérie remonte aux temps les plus reculés [...]. L'apparition de l'Islam a été une révolution globale, humaine dans sa démarche, universelle dans ses principes, arabe dans son expression. [...] Le peuple algérien est un peuple arabe et musulman. »

Indépendant des organisations politiques clandestines (Front des forces socialistes [FFS], Parti de l'avant-garde socialiste [PAGS], Parti de la révolution socialiste [PRS]...), le MCB devient en quelques années le symbole mobilisateur opposé à l'arabisation. C'est globalement la position défendue par les nationalistes de l'aile radicale du PPA/MTLD, celle des congressistes à la Soummam en août 1956, celle des intellectuels opposés au régime à l'image des écrivains Kateb Yacine et Mouloud Mammeri. Par son caractère pacifique, par son ambition de militer pour la défense des droits de l'Homme, pour la

4. Le Front de libération nationale.

5. Révolution africaine, $\mathrm{n}^{\mathrm{o}} 1035$ du 23-29 décembre 1983. 
sauvegarde et la promotion de la culture algérienne d'expression aussi bien arabophone que berbérophone tout en s'ouvrant sur les autres cultures et les autres civilisations, etc., le MCB comptera, jusqu'au printemps 1995, parmi ses sympathisants outre les intellectuels traditionnellement critiques à l'égard de la ligne idéologique au pouvoir, des noms d'envergure internationale: des artistes peintres, des chanteurs engagés, des universitaires, des comédiens, des dramaturges, des réalisateurs et metteurs en scène, et aussi de simples citoyens, si bien qu'à chaque anniversaire de la violation des franchises universitaires par les forces de l'ordre, qui correspond au 20 avril 1980, la Kabylie est entièrement paralysée par la grève à laquelle appelle le MCB et que des centaines de milliers de citoyens sortent dans les rues des villes kabyles pour manifester pacifiquement contre la répression et scander des slogans favorables à la liberté d'expression, à la réhabilitation de la dimension berbère de l'Algérie, à la justice sociale... Ces anniversaires sont l'occasion de s'exprimer librement dans des conférences et de se produire à l'université et dans les établissements scolaires de la Kabylie.

Véritables mobilisateurs de citoyens appartenant aux différents courants d'idées politiques en circulation dans la société algérienne, les animateurs du MCB lancent, clandestinement, la revue Tafsut (Le Printemps) pour servir de porte-parole au mouvement et de «support [à] la réflexion et [aux] analyses des intellectuels berbères en matière culturelle [pour] construire une donnée de fait incontournable et irréversible : une culture berbère autonome et tournée vers l'avenir» (Chaker 1989-1990: 32). Beaucoup de ceux qui y écrivaient feront partie des directions nationales de partis politiques à partir d'octobre 1988. Ce sont donc des opposants à la ligne politique au pouvoir. Certains d'entre eux seront incarcérés en 1985 pour avoir créé La Ligue algérienne pour la défense des droits de l'Homme que la Fédération internationale des droits de l'Homme reconnaîtra immédiatement.

Le 23 février 1989, la nouvelle Constitution consacre le multipartisme tout en ignorant la revendication berbère. À partir de cette date, deux principaux partis politiques inscrivent dans leur programme la revendication berbère : le FFS (Front des forces socialistes) et le RCD (Rassemblement pour la culture et la démocratie). Malgré les querelles entre ces deux formations, le MCB mobilise à Alger plus de cinq cent mille personnes devant l'Assemblée populaire nationale le 25 janvier 1990 et obtient, entre autres, l'augmentation aussi bien du volume que de la durée de retransmission et de diffusion de la radio kabyle et l'ouverture d'un département de langue et culture berbères à Tizi-Ouzou .

L'institutionnalisation d'un résumé du journal télévisé dans les principales variantes du berbère d'Algérie (le kabyle, le chaoui, le mozabite, le touareg) est devenue effective à partir de 1991. À la même période, le FFS

6. Un deuxième département sera ouvert à Bejaïa en 1991 et un troisième à Bouira en 2007. 
comme le RCD lancent chacun un journal d'expression kabyle : Amaynut (Le nouveau) pour le premier, et Asalu (Initiateur) pour le second. Édités à Alger avec les caractères latins conformément à l'usage répandu en Kabylie, les deux journaux disparaîtront en quelques mois. Édité à Tizi-Ouzou, l'hebdomadaire Tamurt (Le pays), qui deviendra Le pays publiait, durant ses quelques années d'existence, ses pages centrales en kabyle écrit avec les caractères latins. D'autres expériences de journalisme en kabyle toujours écrit avec les caractères latins ont été tentées : les pages centrales du quotidien national Le Matin, réservé au centre du pays, la revue Izuran (Racines) que dirigeait le romancier Salem Zenia jusqu'à l'an 2003...

Malgré les querelles entre le FFs et le RCD, le MCB, que ce soit au nom des Commissions nationales (proches de FFS) ou de la Coordination nationale des associations culturelles berbères (proche du RCD), mobilise à chaque fois des dizaines, voire des centaines de milliers de manifestants à l'occasion du 20 avril et durant n'importe quelle période de l'année : lorsqu'il s'agit de la berbérité, les populations répondent à la mobilisation sans se soucier de l'origine de l'appel. Ce souci de se mobiliser pour la berbérité, en dépassant les divergences entre le FFS et le RCD, donnera un cachet spectaculaire à la «grève du cartable » en Kabylie durant l'année scolaire 1994-1995. Depuis septembre 1994, et dans une situation économique d'effondrement, un climat politique meurtrier où l'Algérie comptabilise déjà la mort de dizaines de ses meilleurs enfants, les écoles, les universités et les centres de formation professionnelle de Kabylie sont déserts. Dans les innombrables marches de centaines de milliers de citoyens des villes où active le $\mathrm{MCB}$, sur les murs, sur les banderoles écrites dans les trois langues française, arabe et berbère (en tifinagh et/ou en latin, jamais en arabe) les même mots d'ordre, les même slogans : "Pas d'Algérie sans tamazight », "Nous ne sommes pas des Arabes, tamazight di lakul- l'amazigh à l'école », «Révisezl corrigez l'histoire, l'Algérie n'est pas arabe », «tamazight langue nationale et officielle ». L'État, pour la première fois, reconnaît la légitimité de la revendication berbère : devant le Conseil des ministres, le président Liamine Zeroual déclare, le 5 décembre 1994, que «l'amazighité [la berbérité] est une composante incontournable de l'identité et de la personnalité algérienne aux côtés de l'islam et de l'arabité ». Mais, comme à la veille de la guerre pour l'indépendance, le moment est jugé inopportun pour la satisfaire : se débarrasser du terrorisme était la priorité. Les populations restent donc sceptiques et mobilisées malgré les promesses du gouvernement: le souci de ce dernier était, en fait, l'organisation d'une élection présidentielle pour légitimer la lutte contre le terrorisme et pour élire l'Assemblée nationale, les Assemblées régionales et territoriales et revenir de la sorte à la légalité des institutions dissoutes avec la dissolution du Front islamique du salut (FIS), annoncé vainqueur des élections législatives annulées de décembre 1991. 


\section{Constitutionnalisation de la berbérité}

Les accords d'avril 1995 ont porté sur la création d'une institution attachée à la présidence de la République : le Haut Commissariat à l'Amazighité, chargé de «la réhabilitation et la promotion de l'amazighité, en tant que l'un des fondements de l'identité nationale » et de "l'introduction de la langue amazighe dans le système de l'enseignement et de la communication ${ }^{7}$.

À l'issue d'une formation ad hoc d'enseignants de langue berbère durant le mois d'août 1995, la langue berbère est introduite dans le circuit éducatif à titre expérimental. La volonté politique à faire échec à cette opération n'est un secret pour personne : le choix des membres de la composante du HCA (aucun berbérisant connu n'y figure !) cache mal les véritables intentions des sphères officielles du pays : c'est carrément la méthode d'enseignement des langues étrangères qui est proposée (Kahlouche 2000 : 162-163). La qualité des différents «manuels » d'enseignement du berbère est très loin de ce qu'attendent les enseignants de langue berbère en Kabylie qui, livrés à eux-mêmes, se regroupent en association pour s'entraider dans la réalisation de leur mission qui, compte tenu de ces conditions, est beaucoup plus militante que pédagogique.

La constitutionnalisation de la berbérité se limitera au préambule de la Constitution algérienne, amendée en 1996, à l'issue de l'élection présidentielle de novembre 1995. En présentant, dans ce préambule, la berbérité après l'islamité et l'arabité, le législateur produit une contrevérité car, historiquement, le berbère est premier, l'islam et l'arabe sont venus après. Voici l'extrait de ce préambule constitutionnalisant la berbérité de l'Algérie en plus de son islamité et de son arabité : «Aboutissement d'une longue résistance aux agressions menées contre sa culture, ses valeurs et les composantes fondamentales de son identité que sont l'Islam, l'Arabité et l'Amazighité, le $1^{\text {er }}$ Novembre aura solidement ancré les luttes présentes dans le passé glorieux de la nation. » Aucune indication quant au statut constitutionnel de la langue berbère, sinon le communiqué présidentiel $n^{\circ} 2$ de 1995 qui, avec le calcul politicien visant à déposséder la Kabylie de cette revendication, considère le berbère comme le «patrimoine de tous les Algériens ». Même la disposition chronologique des trois composantes de l'identité algérienne est erronée : en plaçant la berbérité après l'arabité et l'islamité, le législateur prend le contre-courant de l'histoire ! C'est comme si l'identité berbère, donc le peuple berbère, se serait rajoutée à l'arabité et à l'islamité de l'Algérie.

7. Article 4 du décret présidentiel no 95-147 du 27 mai 1995. 


\section{Vers la révolte populaire}

Comparées à la nature, à l'ancrage social et à l'ampleur de la revendication berbère, les concessions des pouvoirs publics sont jugées dérisoires, par le caractère facultatif de l'enseignement de la langue berbère à des classes d'examen, biaisée, par la velléité d'adopter les caractères arabes, et calculées : ménager les arabisants, opposants farouches à la berbérité, et faire accepter aux Kabyles la loi portant généralisation de l'emploi de la langue arabe qui, en contradiction totale avec la reconnaissance pluri-identitaire de la nation algérienne, énoncée dans le préambule de la Constitution de 1996, prend effet à partir du 5 juillet 1998, date-anniversaire de la proclamation de l'indépendance de l'Algérie. À l'approche de cette date, le chanteur kabyle L. Matoub, fervent défenseur de la langue berbère, fait sortir un album appelant à l'union autour de la berbérité. Il est assassiné le jeudi 24 juin 1998 : les populations kabyles, en général, et les jeunes en particulier, investissent les rues et détruisent tout ce qui symbolise l'État et l'arabisation. Les appels au calme n'auront aucun effet : la génération dite de Matoub monte au créneau et la fondation qui porte ce nom remplace le rôle mobilisateur du MCB d'avant l'instrumentalisation de ce dernier. À chaque occasion (au jour de l'An berbère qui correspond au 12 janvier, au 20 avril, à l'anniversaire de l'assassinat du Rebelle, le surnom de Matoub, à la sortie du stade 15 juillet d'Alger, où la JSK remportait consécutivement la Coupe de la Confédération africaine de football durant les saisons sportives 2000, 2001 et 2002), des milliers de citoyens répondent à l'appel de cette fondation pour exiger la lumière sur l'assassinat de Matoub et continuer le combat de celui-ci pour la berbérité.

Le $1^{\text {er }}$ janvier 2000, un groupe de militants essentiellement kabyles lance à Paris la berbère télévision, poussant les autorités algériennes à lancer la chaîne de télévision Tamazight fonctionnant comme une copie de la chaînemère, l'ENTV.

Avril 2001. Le lycéen, Massinissa Guermah, est assassiné à l'intérieur de la caserne de la gendarmerie de Béni Douala, village natal de Matoub. Au même moment, des lycéens sont arrêtés à Amizour dans le district de Béjaïa, le second principal département de la Kabylie. Les marches de protestation des lycéens sont réprimées. Massinissa Guermah est traité de « voyou » par les autorités. La Kabylie se soulève contre la hogra (c'està-dire l'injustice et l'humiliation). C'est le début de la révolte kabyle. Partout en Kabylie, les routes sont barrées, les casernes de la gendarmerie isolées et attaquées, les édifices de l'État saccagés. Même les sièges du RCD et du FFS ne sont pas épargnés. Les populations occupent la rue. Les comités de quartiers et de villages installés organisent les populations. En face, la gendarmerie nationale tire sur les manifestants. De jour comme de nuit, la Kabylie du printemps 2001 est donc le théâtre d'affrontements sanglants. De part et d'autre, la haine culmine et les victimes se chiffrent en dizaines. 
Mis en quarantaine, les gendarmes sont secondés par les CRS et les brigades d'intervention spéciale pour récupérer la rue et protéger les édifices de l'État.

Pour gérer cette révolte, les comités de quartiers et de villages se font connaître comme organisation dénommée l'Aarach ${ }^{8}$. Dépassant les clivages entre le RCD et le FFS, l'Aarach, appelé aussi Mouvement citoyen de Kabylie, réussit l'organisation de plusieurs marches pacifiques. Réunis à El-Kseur, à $20 \mathrm{~km}$ de Béjaïa, les membres de cette organisation élaborent une plateforme de quinze points dont le huitième réitère la revendication du statut de langue nationale et officielle au berbère. Explicitée le 11 juin 2001 à Larbaâ Nait Irathen, à $30 \mathrm{~km}$ de Tizi-Ouzou, cette plate-forme, « considérée scellée et non négociable » devait être remise au président M. Abdelaziz Bouteflika, par des délégués du Mouvement citoyen de la Kabylie lors de la marche sur la présidence du jeudi 14 juin 2001, interdite et réprimée.

Alors que de conclave en conclave, le Mouvement citoyen tente de se structurer, une véritable chasse à l'homme est enclenchée pour arrêter les dirigeants du mouvement. Malgré l'arrestation de ses principales têtes pensantes, le mouvement, soutenu par le FFS et le RCD, demeure mobilisateur: d'où le rejet massif des élections pour le renouvellement de l'Assemblée populaire nationale en mai 2002 et la modification de la Constitution pour consacrer la langue berbère «également nationale ».

Malgré les appels à la désobéissance civile nationale lancés dès le début des violences par le FFS, que les précédents scrutins ont donné comme principale formation politique de la Kabylie et dont le leader est l'un des chefs historiques qui ont déclenché la révolution libératrice du pays, la révolte se limitera à la Kabylie. Les raisons évoquées à l'origine des échauffourées sporadiques, durant l'été 2001, sont très loin d'être comparables à celles de la révolte kabyle : pénurie d'eau, routes à goudronner, lignes électriques à réparer, chômage... C'est loin du projet de démocratie participative porté par le Mouvement citoyen de Kabylie. Les autorités centrales ont-elles réussi à circonscrire la révolte à la Kabylie en mettant en avant le point $\mathrm{n}^{\mathrm{o}} 8$ de la plate-forme du Mouvement relatif au statut constitutionnel de la langue berbère ?

Ce sentiment de singularisation est assumé ouvertement par le Mouvement pour l'autonomie/autodétermination de la Kabylie (MAK). Pour l'heure, il est difficile d'évaluer le poids de cette tendance bien que les discours spontanés renferment des signaux de sa présence dans les esprits.

8. Pluriel de l'Aarch qui signifie tribu en kabyle. 
Les réalités sociohistoriques à l'origine de la prise de conscience identitaire dans le milieu kabyle, de son évolution puis de son émergence à la fin du $\mathrm{XX}^{\mathrm{e}}$ siècle en tant que force sociale et politique diamétralement opposée à l'arabisation ainsi que la gestion par les pouvoirs publics algériens de ce dossier, montrent que la revendication kabylophone est, en fait, le versant linguistique du projet de société hérité de l'aile radicale de la génération de militants pour l'indépendance de l'Algérie. Cette revendication est portée par les populations de jeunes générations aspirant à récupérer/défendre/ promouvoir leur langue et à participer à la gestion de leur pays par l'instauration d'une démocratie participative.

Faculté des Lettres et Langues, Université Mouloud Mammeri, Tizi-Ouzou.

\section{BIBLIOGRAPHIE}

AсHAB, R.

1998 Langue berbère. Introduction à la notation usuelle en caractères latins, Paris, Hoggar.

Ait Ahmed, H.

1983 Mémoire d'un combattant, Paris, Sylvie Messinger.

Ait LARBi, A.

2011 Avril 1980, Alger, Koukou.

Ait SAhlia, A.

1999 La construction de l'identité dans le langage : variations autour de l'identité algérienne, Thèse de doctorat, Rouen, Université de Rouen.

Amrouche, J. M.

1946 «L'Éternel Jugurtha », L’Arche, 13 : 146-164 (réédité dans Algérie, un rêve de fraternité, Paris, Omnibus, 1998).

BASSET, H.

1920 Essai sur la littérature des Berbères, Alger, Ancienne Maison BastilleJourdan.

BenRabah, M.

1996 «L'arabisation des âmes », Linguistique et anthropologie, Cahiers de linguistique sociale, 22 : 13-30.

BOULIFA, S.

1904 Recueil de poésie kabyle, Alger, Adolphe Jourdan.

1897-1913 Méthode de langue kabyle, Alger, Adolphe Jourdan. 


\section{BOUSAFSAF, A.}

1991 «Prise de position des Oulémas musulmans algériens à l'égard du mouvement berbère en Algérie », Revue d'histoire maghrébine, 18 : 243-252.

CHAKer, S.

1981 «De quelques constantes du discours sur les langues populaires en Algérie: de la marginalisation à l'exclusion », Annuaire de l'Afrique du Nord, 20 : 451-457.

1989 «La voie étroite : la revendication berbère entre culture et politique », Annuaire de l'Afrique du Nord, 28 : 281-296.

1989-1990 Imazighen Ass-a, Alger, Bouchène.

1991 Manuel de linguistique berbère 1, Alger, Bouchène.

Cheriet, A.

1983 Opinion sur la politique d'enseignement et l'arabisation, Alger, SNED.

Cortade, J.-M. \& MAmmeri, M.

1967 Lexique français-touareg, Paris, Arts et métiers graphiques.

DESPARMET, J.

1938 «Le panarabisme et la berbérité », Bulletin du Comité de l'Afrique française, Renseignements généraux, 8 \& 9.

Feraoun, M.

1950 Le fils du pauvre, Menrad instituteur kabyle, Lepuy, Cahiers du nouvel humanisme.

1953 La terre et le sang, Paris, Éditions du Seuil.

1957 Les chemins qui montent, Paris, Éditions du Seuil.

1960 Les poèmes de Si Mohand, Paris, Éditions de Minuit.

FICHIER DE DOCUMENTATION BERBÈRE

1946-1976 Fichier de documentation berbère, Fort National.

Granguillaume, G.

1983 Arabisation et politique linguistique au Maghreb, Paris, Maisonneuve \& Larose.

2004 «L'arabisation au Maghreb», Revue de l'Aménagement linguistique, 17 : $15-40$.

Guenoun, A.

1999 Chronologie du mouvement berbère, Alger, Casbah.

HADJAR, A.

1974 «L'arabisation », Asala, 17/18 : 13-27.

HANOTEAU, A.

1858 Essai de grammaire kabyle, Alger, Bastide.

1867 Poésie populaire de la Kabylie du Jurjura, Alger, Librairie A. Jourdan.

1896 Essai de grammaire de la langue tamachek, Paris, Imprimerie impériale. 
HARBI, M.

1992 L'Algérie et son destin. Croyants ou citoyens?, Paris, Arcantère.

2011 [1981] Les archives de la révolution algérienne, Alger, Dahleb.

Kahlouche, R.

2000 «L'enseignement d'une langue non aménagée, au statut indéfini : le berbère en Algérie », Les langues en danger. Mémoires de la société de linguistique de Paris, Nouvelle série, VII : 157-168.

Mammeri, M.

1938 «La société berbère persiste mais ne résiste pas », Aguedal, 5 : 399-412.

1969 Les Isefra, poème de Si Mohand-ou-m’hand, Paris, Maspero.

1976 Tajerrumt $n$ tmazi irit (tantal taqbaylit), Paris, Maspero.

1980a Poèmes kabyles anciens, Paris, Maspero.

1980b Machaho! Contes berbères de Kabylie, Paris, Bordas.

1980c Tellem Chaho! Contes berbères de Kabylie, Paris, Bordas.

1984 L'Ahallil du Gourara, Paris, Éditions de la Maison des sciences de l'homme.

1987 La vie culturelle à Alger 1900-1950, Actes des journées d'études consacrées à « La vie culturelle à Alger», Montpellier, éditeur scientifique P. Siblot.

1988 Précis de grammaire berbère, Paris, Awal.

1989 Inna-yas Ccix Muhend, Alger, Laphomic.

1991 Culture savante, culture vécue : études 1938-1989, Alger, Association scientifique Tala.

MAMMERI, M. (DIR.)

1973 Amawal, tamazit-Tafransist/ Tafransist-Tamazirt, Alger, Imdyazen.

Montassime, A.

1992 Arabisation et politique linguistique au Maghreb, Paris, PUF.

Ouerdane, A.

1990 La question berbère dans le mouvement national algérien 1926-1980, Québec, Sillery ; Paris, Septentrion.

Redjala, R.

1994 «Le long chemin de la revendication berbère », Journal Le pays, 147, 1925 octobre.

SADI, S.

1991 L’Algérie, l'échec recommencé, Alger, Parenthèse.

Salama, A.

1981 L'arabisation en Algérie, Alger, SNED.

SiNI, C.

2007 Contribution à l'analyse des raisons du choix d'une graphie pour écrire le kabyle, Thèse de doctorat, Rouen, Université de Rouen. 
STORA, B.

1985 Ils venaient d'Algérie, Paris, La découverte.

Venture de Paradis, H.

1844 Dictionnaire de langue berbère expliqué (manuscrit volney, bibliothèque F. Mitterrand de Paris, $n^{\circ} 1178$ ).

RÉSUMÉ

Les réalités sociohistoriques à l'origine de la prise de conscience identitaire dans le milieu kabyle, de son évolution puis son émergence à la fin du $X X^{\mathrm{e}}$ siècle en tant que force sociale et politique diamétralement opposée à l'arabisation ainsi que la gestion par les pouvoirs publics algériens de ce dossier, montrent que la revendication kabylophone est, en fait, le versant linguistique du projet de société hérité de I'aile radicale de la génération de militants pour l'indépendance de l'Algérie. Cette revendication est portée par les populations de jeunes générations aspirant à récupérer/ défendre/promouvoir leur langue et à participer à la gestion de leur pays par l'instauration d'une démocratie participative.

\section{ABSTRACT}

The Promotion of Berber in Algeria. From Intellectual Awareness to the Civic Society Project. - The socio-historical realities behind identity awareness in the Kabyle environment, behind its evolution and its eventual emergence in the late twentieth century as a social force are in diametrical opposition to the policy of Arabization and its management by the Algerian public powers. These realities show that the kabylophone claim is, in fact, the linguistic side of the society project inherited from the radical wing of the generation of activists who struggled for the independence of Algeria and which is supported by the younger generations aspiring to recover/ defend/promote their language and participate in the management of their country through the establishment of a participatory democracy.

Mots-clés/Keywords : Algérie, arabisation, constitutionnalisation du berbère, prise de conscience identitaire, radicalisation, revendication culturelle/Algeria, arabization, constitutionalization of Berber, identity awareness, radicalization, cultural claim. 